\title{
A Mixed Nonconforming Finite Element for Linear Elasticity
}

\author{
Zhiqiang Cai, ${ }^{1}$ Xiu $\mathrm{Ye}^{2}$ \\ ${ }^{1}$ Department of Mathematics, Purdue University, West Lafayette, Indiana 47907-1395 \\ ${ }^{2}$ Department of Mathematics and Statistics, University of Arkansas at Little Rock, \\ Little Rock, Arkansas 72204
}

Received 26 February 2005; accepted 2 February 2005

Published online 29 March 2005 in Wiley InterScience (www.interscience.wiley.com).

DOI 10.1002/num.20075

This article considers a mixed finite element method for linear elasticity. It is based on a modified mixed formulation that enforces the continuity of the stress weakly by adding a jump term of the approximated stress on interior edges. The symmetric stress are approximated by nonconforming linear elements and the displacement by piecewise constants. We establish $\mathcal{O}(h)$ error bound in the (broken) $L^{2}$ norm for the divergence of the stress and $\mathcal{O}(h)$ error bound in the $L^{2}$ norm for both the displacement and the stress tensor. @ 2005 Wiley Periodicals, Inc. Numer Methods Partial Differential Eq 21: 1043-1051, 2005

Keywords: elasticity; mixed method; finite element

\section{INTRODUCTION}

Assume that $\Omega \subset \mathfrak{R}^{d}$ ( $d=2$ or 3 ) is a bounded, open, connected domain with Lipschitz boundary $\partial \Omega$. Let $\boldsymbol{\sigma}=\left(\sigma_{i j}\right)_{d \times d}$ be the stress, $\mathbf{u}=\left(u_{1}, \ldots, u_{d}\right)^{t}$ be the displacement, and $\mathbf{f}=$ $\left(f_{1}, \ldots, f_{d}\right)^{t}$ be the body force. Denote by $\boldsymbol{\epsilon}(\mathbf{u})=\left(\epsilon_{i j}(\mathbf{u})\right)_{d \times d}$ the linearized strain tensor with $\epsilon_{i j}(\mathbf{u})=\left(\partial u_{i} / \partial x_{j}+\partial u_{j} / \partial x_{i}\right) / 2, \operatorname{tr}$ the trace operator, and $\nabla \cdot$ the divergence operator. Consider linear elasticity in the stress-displacement formulation:

$$
\begin{cases}\mathcal{A} \boldsymbol{\sigma}-\boldsymbol{\epsilon}(\mathbf{u})=\mathbf{0} & \text { in } \Omega, \\ -\nabla \cdot \boldsymbol{\sigma}=\mathbf{f} & \text { in } \Omega, \\ \mathbf{u}=\mathbf{0} & \text { on } \partial \Omega\end{cases}
$$

where $\mathscr{A}$ is the compliance tensor of fourth order defined by

Correspondence to: Xiu Ye, University of Arkansas at Little Rock, 2801 South University Avenue, Department of Mathematics and Statistics, Little Rock, AR 72204-1099 (e-mail: xxye@ualr.edu)

Contract grant sponsor: National Science Foundation

AMS(MOS) subject classifications: 65F30, 73K25

(๑) 2005 Wiley Periodicals, Inc. 


$$
\mathscr{A} \boldsymbol{\sigma}=\frac{1}{2 \mu}\left(\boldsymbol{\sigma}-\frac{\lambda}{d \lambda+2 \mu}(\operatorname{tr} \boldsymbol{\sigma}) \boldsymbol{\delta}\right)
$$

Here, $\boldsymbol{\delta}=\left(\delta_{i j}\right)_{d \times d}$ is the Kronecker tensor, and positive constants $\lambda$ and $\mu$ are the Lamé constants such that $\mu \in\left[\mu_{1}, \mu_{2}\right]$ with $0<\mu_{1}<\mu_{2}$ and $\lambda \in\left(0, \lambda_{0}\right]$. For simplicity, we assumed homogeneous Dirichlet boundary conditions and finite $\lambda_{0}$. Results in this article can be extended to general boundary conditions and incompressible materials.

There have been many efforts during past four decades to develop stable mixed finite element methods for the system of planar linear elasticity (see [1-11]). Unlike mixed methods for second-order scalar elliptic problems, stress-displacement finite elements are extremely difficult to construct. This is due to the symmetry constraint on the stress tensor. Arnold and Winther in [12] only recently constructed the first family of stable conforming elements in two dimensions on a triangular tessellation. Besides its complication, this family has yet to be extended to three dimensions and to types of nonsimplicial meshes often favored by practitioners. Previous works include two types of approaches. One uses composite elements in which the displacement is approximated on one triangulation of the domain and the stress on a refined triangulation (see $[4-6,11])$. The other is to modify the Hellinger-Reissner variational principle to enforce the symmetry constraint in a weak sense by introducing a Lagrange multiplier or to be abandoned altogether (see [1-3, 8-10, 13]).

The purpose of this article is to study nonconforming linear finite elements of CrouzeixRaviart (CR) [14] for linear elasticity. More specifically, each component of the symmetric stress is approximated by the CR elements and each component of the displacement by piecewise constant elements. This pair is stable and economic, but not convergent. This failure is due to discontinuity of the normal stress on each interior edge. Therefore, we modify the stress-displacement formulation by adding a jump term in the constitutive equation to weakly enforce continuity.

\subsection{Notation}

We use standard notation and definitions for the Sobolev spaces $H^{s}(B)^{d}$, associated inner products $(\cdot, \cdot)_{s, B}$, and respective norms $\|\cdot\|_{s, B}$ and semi-norms $|\cdot|_{s, B}, s \geq 0$. We suppress the designation $d$ because their dependence on dimension is clear by context. We also omit the subscript $B$ from the designation when $B=\Omega$. For $s=0, H^{s}(B)^{d}$ coincides with $L^{2}(B)^{d}$. In this case, the inner product and norm will be denoted by $\|\cdot\|_{B}$ and $(\cdot, \cdot)_{B}$ or $\|\cdot\|$ and $(\cdot, \cdot)$ when $B=\Omega$, respectively. Set

$$
H(\operatorname{div} ; \Omega)=\left\{\mathbf{v} \in L^{2}(\Omega)^{2}: \nabla \cdot \mathbf{v} \in L^{2}(\Omega)\right\}
$$

which is a Hilbert space under the norm

$$
\|\mathbf{v}\|_{H(\operatorname{div} ; \Omega)}=\left(\|\mathbf{v}\|^{2}+\|\nabla \cdot \mathbf{v}\|^{2}\right)^{1 / 2} .
$$

\section{MIXED FINITE ELEMENT APPROXIMATION}

In this section, we introduce a mixed finite element approximation for (1.1). The symmetric stress is approximated by nonconforming piecewise linear elements and the displacement by piecewise constant elements. This pair is stable but not convergent because of its discontinuity 
of the normal stress across interior edges. To enforce the continuity, we add a jump term of the stress in the constitutive equation.

To this end, assume that the domain $\Omega$ is a polygon in two dimensions. Let $\mathscr{T}_{h}$ be a regular triangulation of $\Omega$ with triangular elements of size $O(h)$ (see [15]). Let $\mathscr{E}_{h}$ denote the collection of interior edges of elements in $\mathscr{T}_{h}$. Let $P_{k}(K)$ denote the space of polynomials of degree at most $k \geq 0$ on $K \in \mathfrak{R}^{2}$. The Crouzeix-Raviart nonconforming linear elements [14] are defined as follows:

$$
\mathcal{N} \mathscr{C}^{h}=\left\{v \in L^{2}(\Omega):\left.v\right|_{K} \in P_{1}(K) \forall K \in \mathscr{T}_{h} \text { and } v \text { is continuous at edge midpoints }\right\}
$$

Its degrees of freedom are midpoint values on all edges. For each element $K \in \mathscr{T}_{h}$, denote its three edges by $e_{K}^{i}$ and the corresponding midpoints by $m_{K}^{i}(i=1,2,3)$. There is an interpolation operator $\Pi_{K}: H^{1}(K) \rightarrow P_{1}(K)$ defined by

$$
\Pi_{K} v\left(m_{K}^{i}\right)=\frac{1}{\left|e_{K}^{i}\right|} \int_{e_{K}^{i}} v d \gamma
$$

where $\left|e_{K}^{i}\right|$ denotes the length of the edge $e_{K}^{i}$. It was shown that for all $v \in H^{t}(K)$

$$
\begin{gathered}
\left\|v-\Pi_{K} v\right\|_{K}+h\left|v-\Pi_{K} v\right|_{1, K} \quad C h^{t}|v|_{t, K}, t \in[1,2] \\
\left\|\Pi_{K} v\right\|_{1, K} \quad C\|v\|_{1, K} .
\end{gathered}
$$

The displacement is approximated by piecewise constant vector functions

$$
U^{h}=\left\{\mathbf{v}=\left(\begin{array}{c}
v_{1} \\
v_{2}
\end{array}\right): v_{i} \in L^{2}(\Omega),\left.v_{i}\right|_{K} \in P_{0}(K) \forall K \in \mathscr{T}_{h}, i=1,2\right\}
$$

Let $P_{h}$ be the $L^{2}(\Omega)^{2}$ projection operator onto $U^{h}$, one has the following estimate on each element $K \in \mathscr{T}_{h}$ :

$$
\left\|\mathbf{v}-P_{h} \mathbf{v}\right\|_{K} \quad C h|\mathbf{v}|_{1, K} \quad \forall \mathbf{v} \in H^{1}(K)^{2} .
$$

Each component of the symmetric stress is approximated by the Crouzeix-Raviart element:

$$
\Sigma^{h}=\left\{\boldsymbol{\tau}: \boldsymbol{\tau}^{+}=\boldsymbol{\tau}, \tau_{i j} \in \mathcal{N} \mathscr{C}^{h}, i, j=1,2\right\}
$$

For a symmetric tensor $\boldsymbol{\tau}$, define an interpolation operator $\Pi_{h}$ onto $\Sigma_{h}$ as follows:

$$
\left.\left(\Pi_{h} \tau\right)\right|_{K}=\left(\Pi_{K} \tau_{i j}\right)_{2 \times 2} \quad \forall K \in \mathscr{T}_{h}
$$

It then follows easily from (2.1) and (2.2) that

$$
\left\|\boldsymbol{\tau}-\Pi_{h} \boldsymbol{\tau}\right\|_{K}+h\left|\boldsymbol{\tau}-\Pi_{h} \boldsymbol{\tau}\right|_{1, K} \quad C h^{t}|\boldsymbol{\tau}|_{t, K}, t \in[1,2]
$$




$$
\left\|\Pi_{h} \boldsymbol{\tau}\right\|_{1, K} \quad C\|\boldsymbol{\tau}\|_{1, K}
$$

To define our mixed finite element method, let us first introduce some notations. Let $\mathbf{v} \otimes \mathbf{n}$ denote the matrix whose $i j$ th component is $v_{i} n_{i}$ for two vectors $\mathbf{v}$ and $\mathbf{n}$. For two matrix valued variables $\boldsymbol{\tau}$ and $\boldsymbol{\sigma}$, we define $\boldsymbol{\sigma}: \boldsymbol{\tau}=\sum_{i, j=1}^{2} \boldsymbol{\sigma}_{i j} \boldsymbol{\tau}_{i j}$. Denote the average and jump of scalar, vector tensor functions on an interior edge shared by triangles $K^{+}$and $K^{-}$by

$$
\begin{array}{ll}
\{p\}=\frac{1}{2}\left(p^{+}+p^{-}\right), & {[p]=p^{+} \mathbf{n}^{+}+p^{-} \mathbf{n}^{-},} \\
\{\mathbf{v}\}=\frac{1}{2}\left(\mathbf{v}^{+}+\mathbf{v}^{-}\right), & {[\mathbf{v}]=\mathbf{v}^{+} \cdot \mathbf{n}^{+}+\mathbf{v}^{-} \cdot \mathbf{n}^{-},} \\
\{\boldsymbol{\tau}\}=\frac{1}{2}\left(\boldsymbol{\tau}^{+}+\boldsymbol{\tau}^{-}\right), & {[\boldsymbol{\tau}]=\boldsymbol{\tau}^{+} \cdot \mathbf{n}^{+}+\boldsymbol{\tau}^{-} \cdot \mathbf{n}^{-},}
\end{array}
$$

where $\mathbf{n}^{+}$and $\mathbf{n}^{-}$are unit outward normals to the common edge of the respective $K^{+}$and $K^{-}$. We define a matrix valued jump $\llbracket \rrbracket$ for a vector $\mathbf{w}$ as $\llbracket \mathbf{w} \rrbracket=\mathbf{w}^{+} \otimes \mathbf{n}^{+}+\mathbf{w}^{-} \otimes \mathbf{n}^{-}$.

Define bilinear and linear forms as follows:

$$
\begin{gathered}
a_{s}(\boldsymbol{\sigma}, \boldsymbol{\tau})=(\mathscr{A} \boldsymbol{\sigma}, \boldsymbol{\tau})+\alpha \sum_{e \in \mathscr{C}_{h}} \int_{e} h^{s}[\boldsymbol{\sigma}] \cdot[\boldsymbol{\tau}] d \gamma \quad \forall \boldsymbol{\sigma}, \boldsymbol{\tau} \in \Sigma^{h}, \\
b(\boldsymbol{\tau}, \mathbf{v})=\sum_{K \in \mathscr{T}_{h}}(\mathbf{v}, \nabla \cdot \boldsymbol{\tau})_{K} \quad \forall \boldsymbol{\tau} \in \Sigma^{h}, \forall \mathbf{v} \in U^{h}, \text { and } \quad f(\mathbf{v})=-(\mathbf{f}, \mathbf{v}) \quad \forall \mathbf{v} \in U^{h},
\end{gathered}
$$

where $\alpha$ is a positive constant and $s \in[-1,1]$. Now, the mixed finite element approximation to (1.1) is then to seek $\left(\boldsymbol{\sigma}^{h}, \mathbf{u}^{h}\right) \in \Sigma^{h} \times U^{h}$ such that

$$
\begin{cases}a_{s}\left(\boldsymbol{\sigma}^{h}, \boldsymbol{\tau}\right)+b\left(\boldsymbol{\tau}, \mathbf{u}^{h}\right)=0 & \forall \boldsymbol{\tau} \in \Sigma^{h}, \\ b\left(\boldsymbol{\sigma}^{h}, \mathbf{v}\right)=f(\mathbf{v}) & \forall \mathbf{v} \in U^{h}\end{cases}
$$

Let $\nabla_{h} \cdot \mathbf{v}$ be an $L^{2}(\Omega)$ function whose restriction to each $K \in \mathscr{T}_{h}$ is given by $\left.\nabla \cdot \mathbf{v}\right|_{K}$. For any $\boldsymbol{\tau} \in H(\operatorname{div} ; \Omega)^{2}$ and any $\mathbf{v} \in U^{h}$, it follows from Gauss Divergence Theorem and the definition of the operator $\Pi_{h}$ that

$$
b\left(\boldsymbol{\tau}-\Pi_{h} \boldsymbol{\tau}, \mathbf{v}\right)=\sum_{K \in \mathcal{T}_{h}} \mathbf{v}_{K} \cdot \int_{K} \nabla \cdot\left(\boldsymbol{\tau}-\Pi_{h} \boldsymbol{\tau}\right) d x=\sum_{K \in \mathscr{T}_{h}} \mathbf{v}_{K} \cdot \int_{\partial K}\left(\boldsymbol{\tau}-\Pi_{h} \boldsymbol{\tau}\right) \mathbf{n} d \gamma=0 .
$$

That is

$$
b\left(\boldsymbol{\tau}-\Pi_{h} \boldsymbol{\tau}, \mathbf{v}\right)=0 \quad \forall \mathbf{v} \in U^{h},
$$

which implies the commutativity property:

$$
P_{h} \nabla_{h} \cdot \boldsymbol{\tau}=\nabla_{h} \cdot \Pi_{h} \boldsymbol{\tau} \quad \forall \boldsymbol{\tau} \in H(\operatorname{div} ; \Omega)^{2}
$$


Remark 2.1. The second equation in (2.6) gives local "conservation":

$$
\int_{\partial K} \boldsymbol{\sigma}^{h} \mathbf{n} d \gamma=\int_{K} \nabla \cdot \boldsymbol{\sigma}^{h} d x=-\int_{K} \mathbf{f} d x \quad \forall K \in \mathscr{T}_{h} .
$$

If the body force $\mathbf{f}$ is piecewise constant with respect to the triangulation $\mathscr{T}_{h}$, then the finite element approximation satisfies the equilibrium equation exactly at each element; i.e.,

$$
\nabla_{h} \cdot \boldsymbol{\sigma}^{h}=-\mathbf{f} \quad \text { in } K \in \mathscr{T}_{h}
$$

since the divergence of the stress is piecewise constant.

\section{ERROR ESTIMATION}

Let $(\boldsymbol{\sigma}, \mathbf{u})$ be the exact solution of (1.1) and it is well known that $\mathbf{u}$ and $\boldsymbol{\sigma} \mathbf{n}$ are continuous in the domain $\bar{\Omega}$. For any $\tau \in \Sigma^{h}$, symmetry of $\tau$ and integration by parts give

$$
(\boldsymbol{\epsilon}(\mathbf{u}), \boldsymbol{\tau})=(\nabla \mathbf{u}, \boldsymbol{\tau})=-\sum_{K \in \mathscr{T}_{h}}(\mathbf{u}, \nabla \cdot \boldsymbol{\tau})_{K}+\sum_{K \in \mathscr{T}_{h}} \int_{\partial K} \mathbf{u} \cdot(\boldsymbol{\tau} \mathbf{n}) d \gamma
$$

Using notations of the average and jump and the continuity of $\mathbf{u}$, edge integral terms in the above equalities may be rewritten as

$$
\sum_{K \in \mathscr{T}_{h}} \int_{\partial K} \mathbf{u} \cdot(\boldsymbol{\tau} \mathbf{n}) d \gamma=\sum_{e \in \mathscr{E}_{h} \cup \partial \Omega} \int_{e}(\{\boldsymbol{\tau}\}: \llbracket \mathbf{u} \rrbracket+\{\mathbf{u}\} \cdot[\boldsymbol{\tau}]) d \gamma=\sum_{e \in \mathscr{E}_{h}} \int_{e}\{\mathbf{u}\} \cdot[\boldsymbol{\tau}] d \gamma
$$

The continuity of $\boldsymbol{\sigma}$ yields $[\boldsymbol{\sigma}]=0$ on each interior edge of $\mathscr{T}_{h}$. Hence, $a_{s}(\boldsymbol{\sigma}, \boldsymbol{\tau})=(\mathscr{A} \boldsymbol{\sigma}, \boldsymbol{\tau})$. It is then easy to see that the exact solution, $(\boldsymbol{\sigma}, \mathbf{u})$, satisfies the following equations:

$$
\begin{cases}a_{s}(\boldsymbol{\sigma}, \boldsymbol{\tau})-\sum_{e \in \mathscr{\mathscr { C }}_{h}} \int_{e}\{\mathbf{u}\} \cdot[\boldsymbol{\tau}] d \gamma+b(\boldsymbol{\tau}, \mathbf{u})=0 & \forall \boldsymbol{\tau} \in \Sigma^{h}, \\ b(\boldsymbol{\sigma}, \mathbf{v})=f(\mathbf{v}) & \forall \mathbf{v} \in U^{h} .\end{cases}
$$

Difference of (3.1) and (2.6) gives the following error equations:

$$
\begin{cases}a_{s}\left(\boldsymbol{\sigma}-\boldsymbol{\sigma}^{h}, \boldsymbol{\tau}\right)-\sum_{e \in \mathscr{E}_{h}} \int_{e}\{\mathbf{u}\} \cdot[\boldsymbol{\tau}] d \gamma+b\left(\boldsymbol{\tau}, \mathbf{u}-\mathbf{u}^{h}\right)=0 & \forall \boldsymbol{\tau} \in \Sigma^{h}, \\ b\left(\boldsymbol{\sigma}-\boldsymbol{\sigma}^{h}, \mathbf{v}\right)=0 & \forall \mathbf{v} \in U^{h} .\end{cases}
$$

Denote 


$$
\|\boldsymbol{\tau}\|_{s}=\sqrt{\alpha \sum_{e \in \mathscr{E}_{h}} \int_{e}[\boldsymbol{\tau}]^{2} d \gamma}
$$

for a tensor and

$$
\|\| \mathbf{v} \|_{-s}=\sqrt{\frac{1}{\alpha} \sum_{e \in \mathscr{C}_{h}} h^{-s} \int_{e}\{\mathbf{v}\}^{2} d \gamma}
$$

for a vector. We will need the following estimations.

Lemma 3.1. For any $\tau \in H^{t}(\Omega)^{4}$ with $t \in[1,2]$, one has

$$
\left\|\left.\left|\boldsymbol{\tau}-\Pi_{h} \boldsymbol{\tau}\|\|_{s} \quad C h^{t+(s-1 / 2)}\right| \boldsymbol{\tau}\right|_{t}\right.
$$

For any $\mathbf{v} \in H^{1}(\Omega)^{2}$, one has

$$
\left\|\mathbf{v}-P_{h} \mathbf{v}\right\|_{-s} \quad C h^{(1-s / 2)}|\mathbf{v}|_{1} .
$$

Proof. (3.3) follows easily from the trace theorem and (2.4) that

$$
\left\|\left|\boldsymbol{\tau}-\Pi_{h} \boldsymbol{\tau}\|\|_{s}^{2}=\alpha \sum_{e \in \mathscr{Q}_{h}} h^{s} \int_{e}\left[\boldsymbol{\tau}-\Pi_{h} \boldsymbol{\tau}\right]^{2} d \gamma C \sum_{K \in \mathscr{T}_{h}} h^{s}\left\|\boldsymbol{\tau}-\Pi_{h} \boldsymbol{\tau}\right\|_{K}\right| \boldsymbol{\tau}-\left.\Pi_{h} \boldsymbol{\tau}\right|_{1, K} \quad C h^{2 t+s-1}|\boldsymbol{\tau}|_{t}^{2}\right.
$$

(3.4) follows easily from the trace theorem and (2.3) that

$$
\left\|\left.\left|\mathbf{v}-P_{h} \mathbf{v}\right|\right|_{-s} ^{2}=\frac{1}{\alpha} \sum_{e \in \mathscr{E}_{h}} h^{-s} \int_{e}\left\{\mathbf{v}-P_{h} \mathbf{v}\right\}^{2} d \gamma C \sum_{K \in \mathscr{T}_{h}} h^{-s}\right\| \mathbf{v}-P_{h} \mathbf{v} \|_{K}\left|\mathbf{v}-P_{h} \mathbf{v}\right|_{1, K} \quad C h^{1-s}|\mathbf{v}|_{1}^{2}
$$

(We used the fact that $\left|P_{h} \mathbf{v}\right|_{1, K}=0$ for any $K \in \mathscr{T}_{h}$.) This completes the proof of the lemma. Now, we are ready to establish the following error estimations.

Theorem 3.1. Let $(\boldsymbol{\sigma}, \mathbf{u})$ be the solution of (1.1) and $\left(\boldsymbol{\sigma}^{h}, \mathbf{u}^{h}\right) \in \Sigma^{h} \times U^{h}$ be the solution of (2.6). Then for $\mathbf{f} \in H^{1}(\Omega)^{2}$, we have

$$
\left\|\nabla_{h} \cdot\left(\boldsymbol{\sigma}-\boldsymbol{\sigma}^{h}\right)\right\| \quad C h\|\mathbf{f}\|_{1}
$$

Assume that $(\boldsymbol{\sigma}, \mathbf{u}) \in H^{t}(\Omega)^{4} \times H^{1}(\Omega)^{2}(t \in[1,2])$. Then we have for $\mathbf{f} \in L^{2}(\Omega)^{2}$

$$
\left\|\boldsymbol{\sigma}-\boldsymbol{\sigma}^{h}\right\|_{a_{s}} \quad C\left(h^{t+(s-1 / 2)}|\boldsymbol{\sigma}|_{t}+h^{(1-s / 2)}|\mathbf{u}|_{1}\right)
$$

where $\|\cdot\|_{a_{s}}$ denotes a norm induced from the bilinear form $a_{s}(\cdot, \cdot)$. Furthermore, if $(1.1)$ is $H^{2}$-regular, then we have for $\mathbf{f} \in L^{2}(\Omega)^{2}$ 


$$
\left\|\mathbf{u}-\mathbf{u}^{h}\right\| \quad C\left(h^{\min \{1,(3-s / 2)\}}|\mathbf{u}|_{1}+h^{\min \{t+s, t\}}|\boldsymbol{\sigma}|_{t}\right) .
$$

Proof. Since $\nabla_{h} \cdot \Sigma^{h} \subset U^{h}$, the second equation in (2.6) gives

$$
\nabla_{h} \cdot \boldsymbol{\sigma}^{h}=-P_{h} \mathbf{f}
$$

Then the error bound in (3.5) follows easily from the second equation in (1.1) and the approximation property of the $L^{2}$ projection operator $P_{h}$ in (2.3).

Next, we will establish the following estimate:

$$
\left\|\boldsymbol{\sigma}-\boldsymbol{\sigma}^{h}\right\|_{a_{s}}^{2} \quad C\left\|\Pi_{h} \boldsymbol{\sigma}-\boldsymbol{\sigma}\right\|_{a_{s}}^{2}+C\|\| \mathbf{u}-P_{h} \mathbf{u} \|\left.\right|_{-s} ^{2} .
$$

This, together with Lemma 3.1 and (2.4), implies the error bound of $\left\|\boldsymbol{\sigma}-\boldsymbol{\sigma}^{h}\right\|_{a_{\mathrm{s}}}$ in (3.6). To this end, by the commutativity property in (2.8), the second equation of (1.1), and (3.8), we have that

$$
\nabla_{h} \cdot\left(\Pi_{h} \boldsymbol{\sigma}-\boldsymbol{\sigma}^{h}\right)=P_{h} \nabla_{h} \cdot \boldsymbol{\sigma}-\nabla_{h} \cdot \boldsymbol{\sigma}^{h}=-P_{h} \mathbf{f}+P_{h} \mathbf{f}=0 .
$$

Taking $\boldsymbol{\tau}=\Pi_{h} \boldsymbol{\sigma}-\boldsymbol{\sigma}^{h}$ in the first equation of (3.2) and using the orthogonality of nonconforming elements and the Cauchy-Schwarz and triangle inequalities lead to

$$
\begin{aligned}
& a_{s}\left(\boldsymbol{\sigma}-\boldsymbol{\sigma}^{h}, \Pi_{h} \boldsymbol{\sigma}-\boldsymbol{\sigma}^{h}\right)=\sum_{e \in \mathscr{C}_{h}} \int_{e}\{\mathbf{u}\} \cdot\left[\Pi_{h} \boldsymbol{\sigma}-\boldsymbol{\sigma}^{h}\right] d \gamma=\sum_{e \in \mathscr{C}_{h}} \int_{e}\left\{\mathbf{u}-P_{h} \mathbf{u}\right\} \cdot\left[\Pi_{h} \boldsymbol{\sigma}-\boldsymbol{\sigma}^{h}\right] d \gamma \\
& \left\|| \mathbf { u } - P _ { h } \mathbf { u } | | _ { - s } \left|\| \Pi _ { h } \boldsymbol { \sigma } - \boldsymbol { \sigma } ^ { h } | | \| _ { s } \left\|| \mathbf { u } - P _ { h } \mathbf { u } | | _ { - s } \left(\left\|\left|\Pi_{h} \boldsymbol{\sigma}-\boldsymbol{\sigma}\right|\right\|_{s}+\left\|\left|\boldsymbol{\sigma}-\boldsymbol{\sigma}^{h} \|\right|_{s}\right) \frac{1}{2}\left|\left\|\mathbf{u}-P_{h} \mathbf{u} \mid\right\|_{-s}^{2}\right.\right.\right.\right.\right. \\
& +\frac{1}{2}\left|\left\|\Pi_{h} \boldsymbol{\sigma}-\boldsymbol{\sigma}\right\|\right|_{s}^{2}+\left.\left\|\left|\mathbf{u}-P_{h} \mathbf{u}\right|\right\|\right|_{-s}\left\|\boldsymbol{\sigma}-\boldsymbol{\sigma}^{h}\right\|_{s} .
\end{aligned}
$$

Now, it follows from the Cauchy-Schwarz inequality that

$$
\begin{aligned}
& \left\|\boldsymbol{\sigma}-\boldsymbol{\sigma}^{h}\right\|_{a_{s}}^{2}=a_{s}\left(\boldsymbol{\sigma}-\boldsymbol{\sigma}^{h}, \boldsymbol{\sigma}-\Pi_{h} \boldsymbol{\sigma}\right)+a_{s}\left(\boldsymbol{\sigma}-\boldsymbol{\sigma}^{h}, \Pi_{h} \boldsymbol{\sigma}-\boldsymbol{\sigma}^{h}\right)\left\|\boldsymbol{\sigma}-\boldsymbol{\sigma}^{h}\right\|_{a_{s}}\left\|\boldsymbol{\sigma}-\Pi_{h} \boldsymbol{\sigma}\right\|_{a_{s}}+a_{s}(\boldsymbol{\sigma} \\
& \left.-\boldsymbol{\sigma}^{h}, \Pi_{h} \boldsymbol{\sigma}-\boldsymbol{\sigma}^{h}\right)\left\|\boldsymbol{\sigma}-\boldsymbol{\sigma}^{h}\right\|_{a_{s}}\left\{\left\|\boldsymbol{\sigma}-\Pi_{h} \boldsymbol{\sigma}\right\|_{a_{s}}+\|\| \mathbf{u}-P_{h} \mathbf{u}\|\|_{-s}\right\}+\frac{1}{2}\|\| \mathbf{u}-P_{h} \mathbf{u}\left\|\left.\right|_{-s} ^{2}+\frac{1}{2}\right\| \Pi_{h} \boldsymbol{\sigma}-\boldsymbol{\sigma}\|\|_{s}^{2}
\end{aligned}
$$

This implies (3.9).

Finally, we estimate error bound of the displacement in the $L^{2}$ norm by using standard duality argument. Let $(\tilde{\boldsymbol{\sigma}}, \tilde{\mathbf{u}})$ satisfy the elasticity equation in (1.1) with $\mathbf{f}=\mathbf{u}^{h}-\mathbf{u}$; i.e.,

$$
\begin{cases}\mathscr{A} \tilde{\boldsymbol{\sigma}}-\boldsymbol{\epsilon}(\tilde{\mathbf{u}})=\mathbf{0} & \text { in } \Omega, \\ \nabla \cdot \tilde{\boldsymbol{\sigma}}=\mathbf{u}-\mathbf{u}^{h} & \text { in } \Omega, \\ \tilde{\mathbf{u}}=\mathbf{0} & \text { on } \partial \Omega\end{cases}
$$

Multiplying the second equation above by $\mathbf{u}-\mathbf{u}^{h}$, integrating over the domain $\Omega$, and using (2.7) and the Cauchy-Schwarz inequality give

$$
\left\|\mathbf{u}-\mathbf{u}^{h}\right\|^{2}=b\left(\tilde{\boldsymbol{\sigma}}, \mathbf{u}-\mathbf{u}^{h}\right)=b\left(\tilde{\boldsymbol{\sigma}}-\Pi_{h} \tilde{\boldsymbol{\sigma}}, \mathbf{u}-\mathbf{u}^{h}\right)+b\left(\Pi_{h} \tilde{\boldsymbol{\sigma}}, \mathbf{u}-\mathbf{u}^{h}\right)
$$


$=b\left(\tilde{\boldsymbol{\sigma}}-\Pi_{h} \tilde{\boldsymbol{\sigma}}, \mathbf{u}-P_{h} \mathbf{u}\right)+b\left(\Pi_{h} \tilde{\boldsymbol{\sigma}}, \mathbf{u}-\mathbf{u}^{h}\right)\left\|\nabla_{h} \cdot\left(\tilde{\boldsymbol{\sigma}}-\Pi_{h} \tilde{\boldsymbol{\sigma}}\right)\right\|\left\|\mathbf{u}-P_{h} \mathbf{u}\right\|+b\left(\Pi_{h} \tilde{\boldsymbol{\sigma}}, \mathbf{u}-\mathbf{u}^{h}\right)$.

It follows from (3.2) with $\boldsymbol{\tau}=\Pi_{h} \tilde{\boldsymbol{\sigma}}$, the orthogonality of nonconforming elements, the continuity of $\tilde{\boldsymbol{\sigma}} \mathbf{n}$ on interior edges, and the Cauchy-Schwarz inequality that

$$
\begin{aligned}
& b\left(\Pi_{h} \tilde{\boldsymbol{\sigma}}, \mathbf{u}-\mathbf{u}^{h}\right)=-a_{s}\left(\boldsymbol{\sigma}-\boldsymbol{\sigma}^{h}, \Pi_{h} \tilde{\boldsymbol{\sigma}}\right)+\sum_{e \in \mathscr{E}_{h}} \int_{e}\{\mathbf{u}\} \cdot\left[\Pi_{h} \tilde{\boldsymbol{\sigma}}\right] d \gamma=-a_{s}\left(\boldsymbol{\sigma}-\boldsymbol{\sigma}^{h}, \Pi_{h} \tilde{\boldsymbol{\sigma}}\right) \\
& \quad+\sum_{e \in \mathscr{C}_{h}} \int_{e}\left\{\mathbf{u}-P_{h} \mathbf{u}\right\} \cdot\left[\Pi_{h} \tilde{\boldsymbol{\sigma}}-\tilde{\boldsymbol{\sigma}}\right] d \gamma=-a_{s}\left(\boldsymbol{\sigma}-\boldsymbol{\sigma}^{h}, \Pi_{h} \tilde{\boldsymbol{\sigma}}\right)+\left\|\mathbf{u}-P_{h} \mathbf{u}\right\|\left\|_{-s}\right\| \Pi_{h} \tilde{\boldsymbol{\sigma}}-\tilde{\boldsymbol{\sigma}} \mid \|_{s} .
\end{aligned}
$$

By the definition of $a_{s}(\cdot, \cdot)$, the continuity of $\tilde{\boldsymbol{\sigma}} \mathbf{n}$, and the Cauchy-Schwarz inequality, we have that

$$
\begin{gathered}
a_{s}\left(\boldsymbol{\sigma}-\boldsymbol{\sigma}^{h}, \Pi_{h} \tilde{\boldsymbol{\sigma}}\right)=\left(\mathscr{A}\left(\boldsymbol{\sigma}-\boldsymbol{\sigma}^{h}\right), \Pi_{h} \tilde{\boldsymbol{\sigma}}-\tilde{\boldsymbol{\sigma}}\right)+\left(\mathscr{A}\left(\boldsymbol{\sigma}-\boldsymbol{\sigma}^{h}\right), \tilde{\boldsymbol{\sigma}}\right)+\alpha \sum_{e \in \mathscr{E}_{h}} \int_{e} h^{s}\left[\boldsymbol{\sigma}-\boldsymbol{\sigma}^{h}\right] \cdot\left[\Pi_{h} \tilde{\boldsymbol{\sigma}}\right. \\
-\tilde{\boldsymbol{\sigma}}] d \gamma C\left\|\boldsymbol{\sigma}-\boldsymbol{\sigma}^{h \|}\right\| \Pi_{h} \tilde{\boldsymbol{\sigma}}-\tilde{\boldsymbol{\sigma}}\|+\| \boldsymbol{\sigma}-\boldsymbol{\sigma}^{h}\|\|_{s}\left\|\Pi_{h} \tilde{\boldsymbol{\sigma}}-\tilde{\boldsymbol{\sigma}}\right\|_{s}+\left(\mathscr{A} \tilde{\boldsymbol{\sigma}}, \boldsymbol{\sigma}-\boldsymbol{\sigma}^{h}\right) .
\end{gathered}
$$

Multiplying the first equation of (3.11) by $\boldsymbol{\sigma}-\boldsymbol{\sigma}^{h}$, integrating over the domain $\Omega$, and using integration by parts lead to

$$
\left(\mathscr{A} \tilde{\boldsymbol{\sigma}}, \boldsymbol{\sigma}-\boldsymbol{\sigma}^{h}\right)+b\left(\boldsymbol{\sigma}-\boldsymbol{\sigma}^{h}, \tilde{\mathbf{u}}\right)-\sum_{e \in \mathscr{E}_{h}} \int_{e}\{\tilde{\mathbf{u}}\} \cdot\left[\boldsymbol{\sigma}-\boldsymbol{\sigma}^{h}\right] d \gamma=0
$$

This, together with the second equation of (3.2), the orthogonality of nonconforming elements, (3.10), and the Cauchy-Schwarz inequality, implies

$$
\begin{gathered}
\left(\mathscr{A}\left(\boldsymbol{\sigma}-\boldsymbol{\sigma}^{h}\right), \tilde{\boldsymbol{\sigma}}\right)=b\left(\boldsymbol{\sigma}-\boldsymbol{\sigma}^{h}, P_{h} \tilde{\mathbf{u}}-\tilde{\mathbf{u}}\right)+\sum_{e \in \mathscr{E}_{h}} \int_{e}\left\{\tilde{\mathbf{u}}-P_{h} \tilde{\mathbf{u}}\right\} \cdot\left[\boldsymbol{\sigma}-\boldsymbol{\sigma}^{h}\right] d \gamma \\
=b\left(\boldsymbol{\sigma}-\prod_{h} \boldsymbol{\sigma}, P_{h} \tilde{\mathbf{u}}-\tilde{\mathbf{u}}\right)+\sum_{e \in \mathscr{E}_{h}} \int_{e}\left\{\tilde{\mathbf{u}}-P_{h} \tilde{\mathbf{u}}\right\} \cdot\left[\boldsymbol{\sigma}-\boldsymbol{\sigma}^{h}\right] d \gamma\left\|\nabla_{h} \cdot\left(\boldsymbol{\sigma}-\Pi_{h} \boldsymbol{\sigma}\right)\right\|\left\|P_{h} \tilde{\mathbf{u}}-\tilde{\mathbf{u}}\right\| \\
+\left\|\tilde{\mathbf{u}}-P_{h} \tilde{\mathbf{u}}\right\|\left\|_{-s}\right\| \boldsymbol{\sigma}-\boldsymbol{\sigma}^{h}\|\|_{s} .
\end{gathered}
$$

Combining inequalities (3.12)-(3.15) and using Lemma 3.1 and (3.6), we have that 


$$
\begin{array}{r}
\left\|\mathbf{u}-\mathbf{u}^{h}\right\|^{2} \quad\left\|\nabla_{h} \cdot\left(\tilde{\boldsymbol{\sigma}}-\Pi_{h} \tilde{\boldsymbol{\sigma}}\right)\right\|\left\|\mathbf{u}-P_{h} \mathbf{u}\right\|+\left\|\left|\mathbf{u}-P_{h} \mathbf{u}\left\|\left.\right|_{-s}\right\| \Pi_{h} \tilde{\boldsymbol{\sigma}}-\tilde{\boldsymbol{\sigma}}\|\|_{s}+C\left\|\boldsymbol{\sigma}-\boldsymbol{\sigma}^{h}\right\| \| \Pi_{h} \tilde{\boldsymbol{\sigma}}\right.\right. \\
-\tilde{\boldsymbol{\sigma}}\|+\| \boldsymbol{\sigma}-\left.\boldsymbol{\sigma}^{h}\|\|_{s}\left\|\Pi_{h} \tilde{\boldsymbol{\sigma}}-\tilde{\boldsymbol{\sigma}}\left|\left\|_{s}+\right\| \nabla_{h} \cdot\left(\boldsymbol{\sigma}-\Pi_{h} \boldsymbol{\sigma}\right)\|\| P_{h} \tilde{\mathbf{u}}-\tilde{\mathbf{u}}\|+\| \tilde{\mathbf{u}}-P_{h} \tilde{\mathbf{u}}\right|\right\|\right|_{-s} \| \mid \boldsymbol{\sigma} \\
-\boldsymbol{\sigma}^{h}\|\|_{s} C\left\{h\|\tilde{\boldsymbol{\sigma}}\|_{1}|\mathbf{u}|_{1}+h^{(1-s / 2)}|\mathbf{u}|_{1} h^{(1+s / 2)}|\tilde{\boldsymbol{\sigma}}|_{1}+\left(h^{t+(s-1 / 2)}|\boldsymbol{\sigma}|_{t}+h^{(1-s / 2)}|\mathbf{u}|_{1}\right)\left(h|\tilde{\boldsymbol{\sigma}}|_{1}+h^{(s+1 / 2)}|\tilde{\boldsymbol{\sigma}}|_{1}\right)\right. \\
\left.+h^{t-1}|\boldsymbol{\sigma}|_{t} h|\tilde{\mathbf{u}}|_{1}+h^{(1-s / 2)}|\tilde{\mathbf{u}}|_{1} h^{t+(s-1 / 2)}|\boldsymbol{\sigma}|_{t}\right\} .
\end{array}
$$

Now, (3.7) follows from the $H^{2}$ regularity estimate: $\|\tilde{\mathbf{u}}\|_{2}+\|\tilde{\boldsymbol{\sigma}}\|_{1} \quad C\left\|\mathbf{u}-\mathbf{u}^{h}\right\|$. This completes the proof of the theorem.

\section{References}

1. M. Amara and J. M. Thomas, Equilibrium finite elements for the linear elasticity problem, Numer Math 33 (1979), 367-383.

2. D. N. Arnold and R. S. Falk, A new mixed formulation for elasticity, Numer Math 53 (1988), $13-30$.

3. D. N. Arnold, F. Brezzi, and J. Douglas, Jr., PEERS: A new mixed finite element for plane elasticity, Jpn J Appl Math 1 (1984), 347-367.

4. D. N. Arnold, J. Douglas, Jr., and C. P. Gupta, A family of higher order mixed finite element methods for plane elasticity, Numer Math 45 (1984), 1-22.

5. B. X. Fraeijs de Veubeke, Displacement and equilibrium models in the finite element method, O. C. Zienkiewics and G. S. Holister, editors, Stress analysis, Wiley, New York, 1965, pp. 145-197.

6. C. Johnson and B. Mercier, Some equilibrium finite element methods for two-dimensional elasticity problems, Numer Math 30(1) (1978), 103-116.

7. A. L. Mignot and C. Surry, A mixed finite element family in plane elasticity, Appl Math Modelling 5 (1981), 259-262.

8. E. Stein and R. Rolfes, Mechanical conditions for stability and optimal convergence of mixed finite elements for linear plane elasticity, Comput Methods Appl Mech Engrg 84(1) (1990), 77-95.

9. R. Stenberg, On the construction of optimal mixed finite element methods for the linear elasticity, Numer Math 48(4) (1986), 447-462.

10. R. Stenberg, A family of mixed finite elements for the elasticity problem, Numer Math 53 (1988), 513-538.

11. V. B. Watwood, Jr. and B. J. Hartz, An equilibrium stress field model for finite element solution of two-dimensional elastostatic problems, Internat Jour Solids Struct 4 (1968), 857-873.

12. D. N. Arnold and R. Winther, Mixed finite elements for elasticity, Numer Math (2001), to appear.

13. R. Stenberg, Two low-order mixed methods for the elasticity problem, The mathematics of finite elements and applications, Vol. VI (Uxbridge, 1987), Academic Press, London, 1988, pp. 271-280.

14. M. Crouzeix and P. A. Raviart, Conforming and nonconforming finite element methods for solving the stationary Stokes equations, RAIRO Anal Numer 7 (1973), 33-76.

15. P. G. Ciarlet, The finite element method for elliptic problems, North-Holland, New York, 1978. 